\title{
Temporal frequency discrimination in amblyopia
}

\author{
XUBO YANG ${ }^{1,2^{*}}$, JIHONG ZENG $^{1 *}$, JIANGLAN WANG ${ }^{2}$ and LONGQIAN LIU ${ }^{1,2}$ \\ Departments of ${ }^{1}$ Ophthalmology, and ${ }^{2}$ Optometry and Visual Science, \\ West China Hospital of Sichuan University, Chengdu, Sichuan 610041, P.R. China
}

Received March 20, 2018; Accepted August 28, 2018

DOI: $10.3892 /$ etm.2018.6688

\begin{abstract}
The present study aimed to explore temporal frequency discrimination in amblyopia, and the influence of attentional load on amblyopic temporal cognition. Twenty anisometropic amblyopes, 20 strabismic amblyopes and 20 normal subjects were recruited. A flickering disk was used for visual stimuli. A two-alternative, forced-choice staircase paradigm was used to measure the temporal frequency discrimination thresholds of amblyopic eyes, fellow eyes and normal dominant eyes under reference temporal frequencies of 5,10 and $20 \mathrm{~Hz}$. The just-noticeable differences data were analyzed statistically. In addition, the temporal frequency discrimination thresholds of the subjects were measured under different conditions of attentional load. The changes in temporal frequency discrimination thresholds were compared between amblyopic and normal eyes when attention was loaded. At the reference temporal frequencies of 5 and $10 \mathrm{~Hz}$, temporal thresholds of amblyopic eyes were significantly increased compared with fellow eyes $(\mathrm{P}<0.05)$. Regarding strabismic amblyopia, temporal thresholds of fellow eyes were increased compared with normal eyes $(\mathrm{P}<0.05)$. However, no correlation was identified between the temporal-frequency discrimination thresholds and the LogMAR visual acuities in amblyopic eyes. In addition, higher attentional load caused greater changes in temporal thresholds in amblyopic eyes, but the situation was not the same for lower attentional load. In conclusion, in the present study a deficit of temporal frequency discrimination in amblyopic eyes and the fellow eyes of strabismic amblyopes was identified. Furthermore, the influence of attentional load on temporal frequency discrimination in amblyopic eyes was increased compared with normal eyes.
\end{abstract}

Correspondence to: Professor Longqian Liu, Department of Opthalmology, West China Hospital of Sichuan University, 37 Guoxue Xiang, Chengdu, Sichuan 610041, P.R. China

E-mail: b.q15651@hotmail.com

*Contributed equally

Key words: amblyopia, temporal frequency discrimination, attentional load

\section{Introduction}

Amblyopia is a commonly occurring disease of abnormal visual development characterized by a decrease in best-corrected visual acuity without any ocular organic disease. It is typified by deficient spatial vision, such as loss of central vision, resulting in a reduced spatial contrast sensitivity function (CSF). Visuotemporal deficits are also associated with amblyopia (1). To date, there are four methods of evaluating temporal processing: Time reproduction, time production, verbal estimation and time comparison. Certain scholars consider that time comparison, particularly temporal discrimination, is the purest and best method of measuring time perception and short time differences compared with time reproduction and time production (2).

Studies published on temporal discrimination in amblyopia are rare. Spang and Fahle (3), suggested that the temporal discrimination of amblyopia deteriorated based on a task of time-based, figure-ground segregation. However, whether this deterioration potentially originated from either lower-level temporal processing deficits or higher-level figure-ground segregation deficits could not be clarified. In addition, earlier studies demonstrated that the visual perception of amblyopia was influenced by attention using tasks of object tracking (4-7), object enumeration (8), attentional blink (9), decision making (10), and line bisection (11). Recently, Hou et al (12) identified the degraded attentional modulation of cortical neural populations in strabismic amblyopia. Furthermore, attention was involved in the process of temporal cognition for normal subjects $(13,14)$. However, how attentional load influences temporal cognition in amblyopia has yet to be fully elucidated.

The aim of the present study was to determine whether there is a deficit of temporal frequency discrimination in amblyopia, and to determine the influences of attentional load on amblyopic temporal discrimination.

\section{Patients and methods}

Subjects. The present study enrolled 20 individuals with anisometropic amblyopia, 20 individuals with strabismic amblyopia, and 20 normal subjects who were aged 10-14 years between February 1, 2015 and January 31, 2016. Any subjects who had a history of neurological dysfunction, such as attention deficit hyperactivity disorder (ADHD), or had ocular diseases other than amblyopia, or were not able to cooperate were excluded. 
Full optical correction was required during the present study. All subjects were naive to the purposes of the experiment. The study was performed in accordance with the Declaration of Helsinki, and all participants' guardians signed written informed consent. The experimental protocol was approved by the institutional review board of West China Hospital of Sichuan University (Chengdu, China). All information pertaining to the subjects was anonymized and identified prior to analysis.

Visual stimulus. The stimulus featured a white square-wave flickery disc against a black background (the contrast was set at $96 \%$ ). The disc, which was shown at a central position on the screen, subtended an arc of $\sim 1^{\circ}$ at a viewing distance of $\sim 1 \mathrm{~m}$. There was a black fixation cross in the middle of the disc that did not flicker with the disc. The flicker frequency and the flicker duration exhibited an association of inverse function. The stimulus was displayed on a 19-inch Samsung CRT computer monitor at a resolution of $1024 \times 768$ pixels, and at an $85-\mathrm{Hz}$ refresh rate.

Procedural details. Prior to performing the temporal discrimination task, the subjects received a general ocular examination, including a LogMAR visual acuity test and refraction. Subsequently, the participants viewed the screen with their tested eyes at a distance of $\sim 1 \mathrm{~m}$ in a darkened room. The temporal discrimination task was tested at the reference frequency of 5,10 , or $20 \mathrm{~Hz}$. The fellow eye was tested first, and then the amblyopic eye.

A two-alternative, forced-choice (2AFC) task procedure, a classical method of measuring frequency discrimination (15), was performed in the present study. Each trial consisted of two $800 \mathrm{~ms}$ intervals separated by $600 \mathrm{~ms}$. One interval contained the flickery disk of the reference temporal frequency, whereas the other included the comparison temporal frequency. The sequence of each was random. Subjects were required to respond using a keyboard. Pressing the ' $\leftarrow$ ' key suggested that the first interval of the disk flickered faster compared with the second, whereas pressing the ' $\rightarrow$ ' key indicated the opposite. Regarding the comparison temporal frequency, the start value of flicker duration was set at $30 \%$ of the reference flicker duration. Pilot experiments indicated that these values were discriminable. The step size of the staircase was $10 \%$ of the reference flicker duration. The comparison flicker duration varied initially using an ascending 1-up, 1-down staircase. When an incorrect response was recorded, staircases changed to an ascending 3-up, 1-down staircase. These staircases ended after seven reversals; therefore, the numbers of trials per run varied. Just-noticeable differences (JNDs) were calculated by taking the geometric mean of the flicker duration differences between the reference and comparison flicker duration for the last four reversals of each staircase. JNDs of the amblyopic eye, fellow eye and normal eye were tested in the present study, and these represented the thresholds of temporal discrimination.

Attentional load was fulfilled according to the classical experimental paradigm of double tasks (16). Here, doing mental arithmetic when testing temporal discrimination was used for double tasks. When the participant was required to respond by pressing the keyboard, an arithmetic equation was shown at the top of the screen. The participant was required to perform the mental arithmetic and read the result aloud. This action would occupy the working memory capacity, and divert attention towards the task that was irrelevant to temporal discrimination. Lower attentional load was performed by the sum of ones digits, whereas higher attentional load was performed by the sum of tens digits. JNDs of the amblyopic eye and normal eye were tested under the condition of different attentional loads.

Data analysis. Data analysis was performed using SPSS v.16.0 software (IBM Corp., Armonk, NY, USA) and SAS software, v.8.2 (SAS Institute, Inc., Cary, NC, USA). The age of normal and amblyopic subjects was compared using Student's t-test. The gender of the two subjects was compared using chi-square test. If the data met the normal distribution and homogeneity of variance, JNDs among amblyopic eyes, fellow eyes and normal eyes (dominant eyes) were compared using one-way analysis of variance followed by pairwise comparison using the least significant difference (LSD) method. Otherwise, those data were analyzed using the Kruskal-Wallis nonparametric test, and pairwise comparison was performed using the Nemenyi method featured in the SAS v.8.2 software. The association between amblyopic temporal discrimination and corrected visual acuity was analyzed by Pearson correlation analysis. The changes in JNDs by attentional load between amblyopic and normal eyes were compared using group $\mathrm{t}$-tests. $\mathrm{P}<0.05$ was considered to indicate a statistically significant difference.

\section{Results}

Comparison of demographic parameters between normal and amblyopic subjects. Patients in the amblyopic group included 22 males and 18 females aged $12.1 \pm 1.6$ years. The normal group included 11 males and 9 females aged $12.2 \pm 1.5$ years. Age and gender did not differ significantly between the two groups ( $\mathrm{P}>0.05)$.

Threshold of temporal discrimination. As shown in Fig. 1, at 5 and $10 \mathrm{~Hz}$, temporal thresholds of amblyopic eyes were significantly increased compared with fellow eyes, either for anisometropic amblyopia or for strabismic amblyopia $(\mathrm{P}<0.05)$. No significant differences in the JNDs were identified between fellow eyes and normal eyes for anisometropic amblyopia $(\mathrm{P}>0.05)$. However, for strabismic amblyopia, temporal thresholds of fellow eyes were increased compared with normal eyes $(\mathrm{P}<0.05)$. At $20 \mathrm{~Hz}$, no significant differences in temporal thresholds among these eyes were identified.

Association between temporal discrimination deficit and spatial deficit in amblyopia. No significant correlation between the temporal threshold and LogMAR visual acuity in amblyopia was observed ( $\mathrm{P}>0.05$; Fig. 2).

Changes in temporal thresholds by attentional load in amblyopic and normal eyes. As shown in Fig. 3, at 5 and $10 \mathrm{~Hz}$, lower attentional load evoked no significantly different changes in temporal thresholds between amblyopic and normal eyes. However, higher attentional load caused increased changes in temporal thresholds in amblyopic eyes compared with normal eyes. 

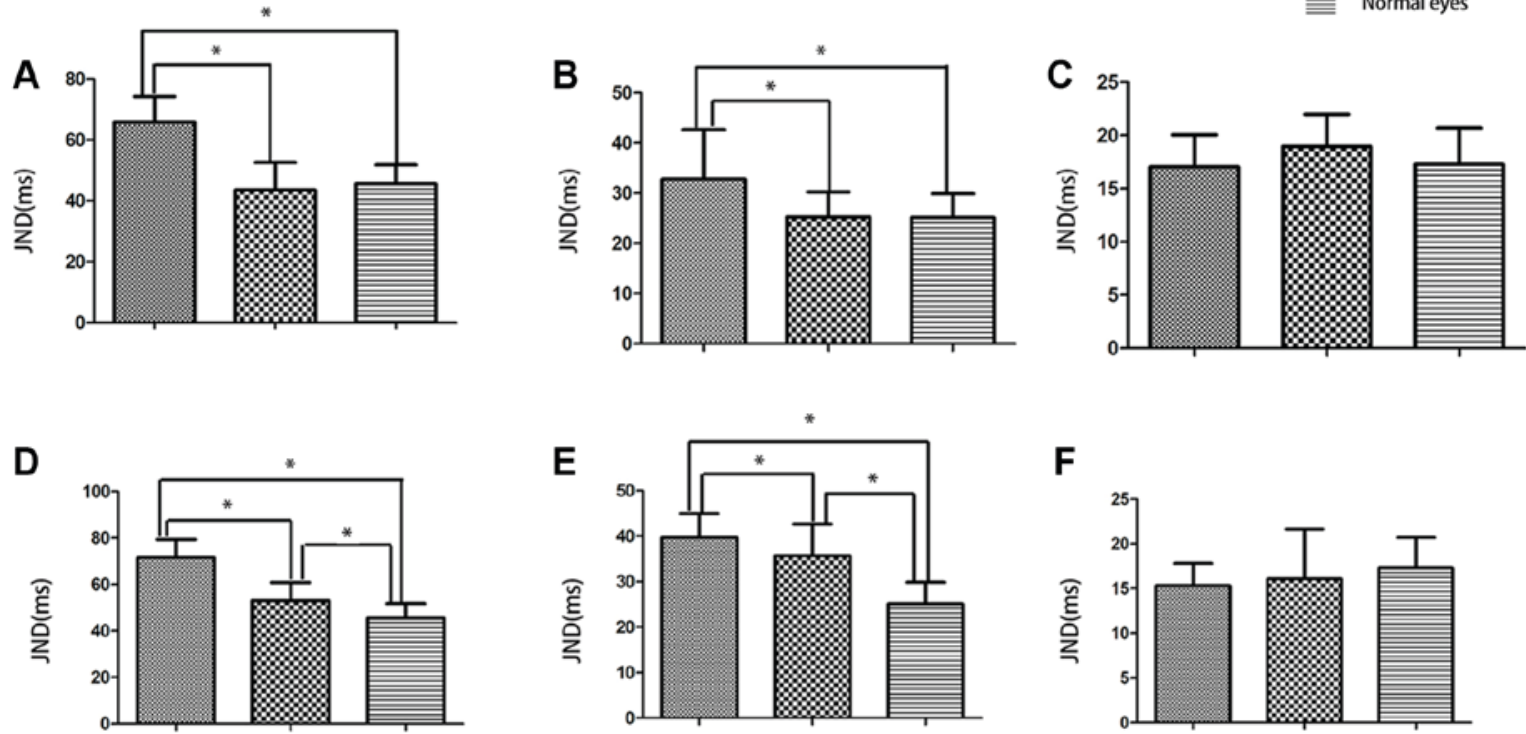

Figure 1. Comparison of JNDs of temporal discrimination among (A-C) anisometropic and (D-F) strabismic amblyopic eyes, fellow eyes and normal eyes JNDs of amblyopia at the reference frequency of (A and D) 5, (B and E) 10, and (C and F) $20 \mathrm{~Hz}$. " $\mathrm{P}<0.05$. JND, just-noticeable difference.

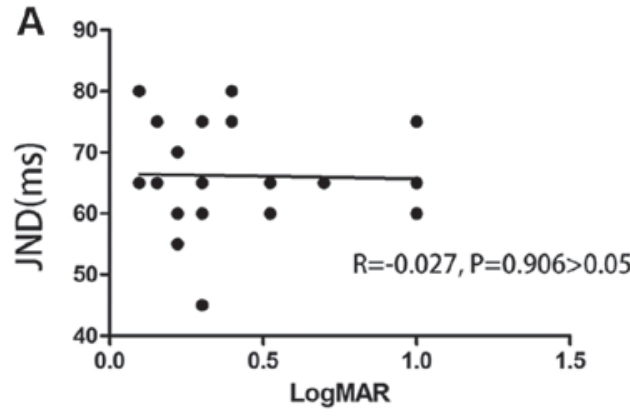

C

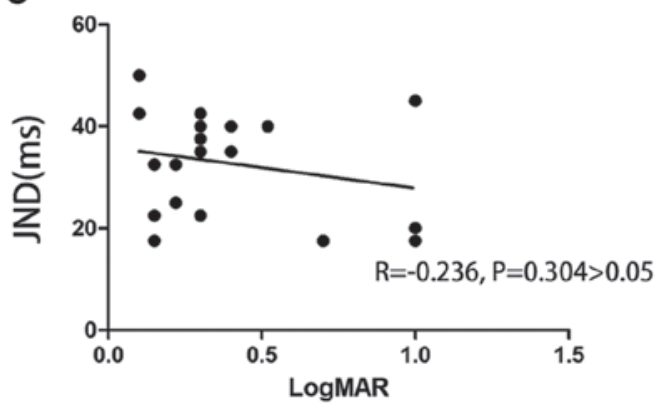

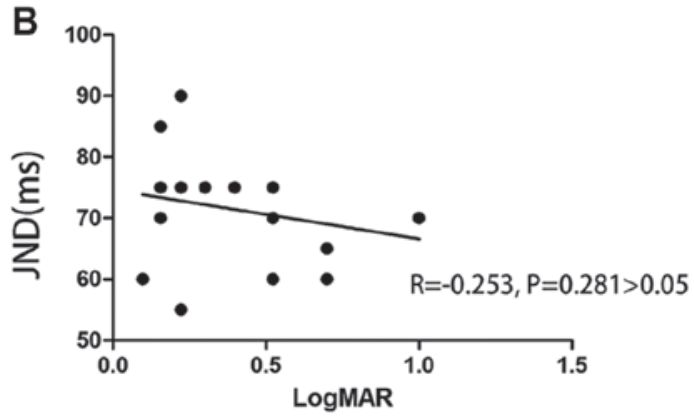

D

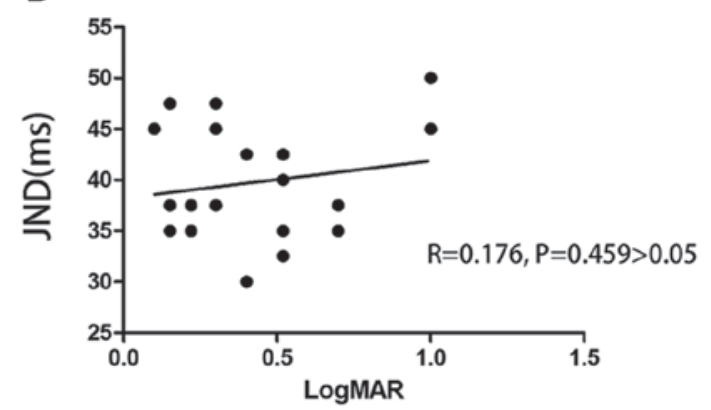

Figure 2. Association between JNDs of temporal discrimination and LogMAR visual acuities in (A and C) anisometropic and (B and D) strabismic amblyopic eyes at the reference frequency of (A and B) 5 and $(\mathrm{C}$ and D) $10 \mathrm{~Hz}$. JND, just-noticeable difference.

\section{Discussion}

Possible mechanism of temporal discrimination deficit in amblyopia. The present study revealed that a larger duration difference for amblyopic eyes compared with normal eyes was required for differentiation to occur at the lower temporal frequencies ( 5 and $10 \mathrm{~Hz}$ ). This finding was consistent with the research results obtained for temporal contrast sensitivity function (TCSF), which was shown to have deteriorated at lower temporal frequencies and be normal at higher temporal frequencies (17). Although the present study focused on the discrimination process and the study investigating TCSF was focused on the detection process, the two studies exhibited similar characteristics, indicating that a common mechanism might exist between temporal detection and temporal discrimination.

Moulden et al (18) reported two channels for flicker in the human system. One channel was a low-pass channel with a peak value of $\sim 5 \mathrm{~Hz}$, whereas the other channel was a band-pass 
A

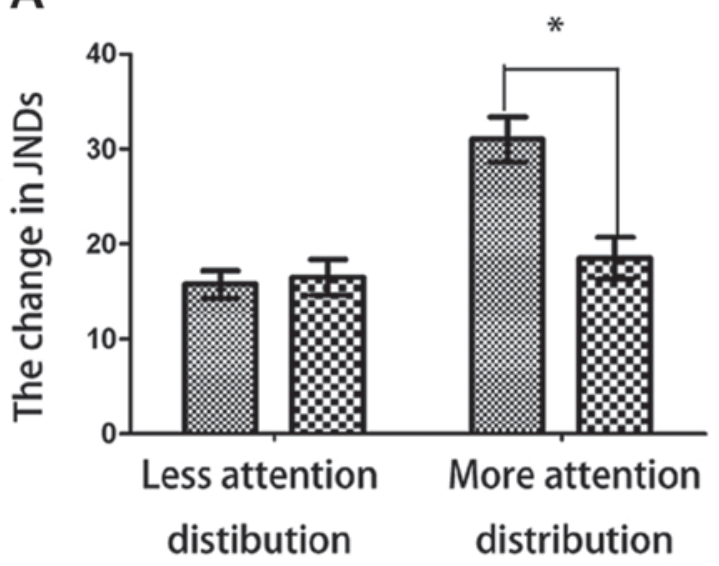

B

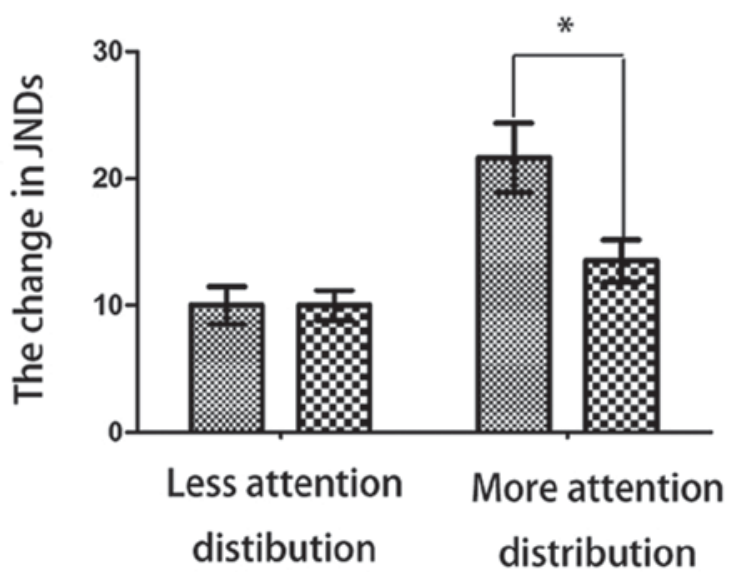

Figure 3. Changes in the temporal-frequency discrimination thresholds caused by attentional load at the reference frequency of (A) 5 and (B) $10 \mathrm{~Hz}$. ${ }^{*}<<0.05$. JND, just-noticeable difference.

channel with a peak value of $\sim 10 \mathrm{~Hz}$. The finding reported in the present study of temporal discrimination declining at 5 and $10 \mathrm{~Hz}$ in amblyopia was similar to the peak values of the two channels. Therefore, it was possible to hypothesize that this finding was associated with the channel positions for the flicker.

Temporal discrimination deficit of fellow eyes in amblyopia and its possible mechanism. Leguire et al (19) proposed that the normal eye of amblyopia was not normal by testing the CSF. Their results were subsequently corroborated using visual evoked potential (20). For the temporal deficits of fellow eyes in amblyopia, numerous studies have evaluated these phenomena, and these deficits were revealed to be most robust for motion perception tasks (21-23). To date, and to the best of our knowledge, the temporal discrimination of the fellow eye in amblyopia based on assigning the task of flickery disk has rarely been reported in the literature. The present study revealed a temporal discrimination deficit in the fellow eye of strabismic amblyopia, but not for anisometropic amblyopia. It is therefore possible that different mechanisms might operate between them.

Association between visuotemporal deficit and visuospatial deficit in amblyopia. Numerous researchers have held different opinions on the association between temporal deficit and spatial deficit in amblyopia. Some considered that the temporal deficit in amblyopia was due to a spatial deficit $(24,25)$. However, other studies suggested that the temporal deficit in amblyopia was independent of the spatial deficit (26). In the present study, the contrast of the flickery stimulus was far beyond the contrast threshold, and its subtended visual angle was within the scope of amblyopic visual acuity. Furthermore, the uniform disk contained the spatial frequency information of the wide band. Thus, our hypothesis was that the temporal discrimination deficit in amblyopia was not caused by a spatial deficit.
In addition, our results suggested that no association existed between the visuotemporal deficit and visual acuities in amblyopia, which was similar to the results identified by Huang et al (26).

Related studies about temporal frequency discrimination in amblyopia. Spang and Fahle (3) suggested that there was a temporal frequency discrimination deficit in amblyopia using the figure-ground segregation paradigm and 4AFC method. However, there were two shortcomings with the stimulus in that study. First, the stimulus could not always maintain central fixation, whereas temporal discrimination was influenced by central fixation and eccentric fixation (26). Secondly, the rotary stimulus included unnecessary information, such as motion, direction and orientation, which might have interfered with the results. In the present study, a flickery disk with a fixation cross was used as the stimulus, which maintained central fixation and contained pure temporal information, without any information regarding motion, direction, and orientation.

Possible mechanism of attentional load affecting temporal discrimination in amblyopia. In the field of study of diseases involving temporal discrimination and attention, ADHD has been the most studied. This attention deficit disease is low in temporal discrimination (27), which is located in the left inferior frontal cortex as assessed by functional magnetic resonance imaging (fMRI) (28). The cognitive process of attention is exclusively associated with the frontal lobe (29). Li et al (30) demonstrated that the white matter volume of the bilateral inferior frontal lobes was lost in amblyopes by voxel-based morphometry of MRI. Thus, the discovery of temporal deficits in amblyopia in the present study may be associated with attention deficit by frontal inactivation. This hypothesis will be investigated further in our future studies.

In conclusion, it was shown in the present study that temporal frequency discrimination is deteriorated in amblyopic eyes and the fellow eyes of strabismic amblyopes. 
Furthermore, the influence of attentional load on temporal frequency discrimination in amblyopic eyes was increased compared with normal eyes.

\section{Acknowledgements}

Not applicable.

\section{Funding}

The present study was supported by Sichuan Science and Technology Program (grant no. 2018SZ0146).

\section{Availability of data and materials}

The datasets used and analyzed during the present study are available from the corresponding author on reasonable request.

\section{Authors' contributions}

$\mathrm{XY}$ and $\mathrm{JZ}$ designed the investigation and drafted the manuscript. JW collected and analyzed the patient data. LL conceptualized the research and revised the manuscript. All authors read and approved the final manuscript.

\section{Ethics approval and consent to participate}

The experimental protocol was approved by the institutional review board of West China Hospital of Sichuan University (Chengdu, China; no. 201433).

\section{Patient consent for publication}

The guardians provided written informed consent for the publication of any associated data.

\section{Competing interests}

The authors declare that they have no competing interests.

\section{References}

1. Yang $X$ and Liu L: Research progress on visual defects of amblyopia. Chin J Exp Ophthalmol 35: 1139-1142, 2017.

2. Rammsayer TH: Ageing and temporal processing of durations within the psychological present. Eur J Cogn Psychol 13 549-565, 2001.

3. Spang K and Fahle M: Impaired temporal, not just spatial, resolution in amblyopia. Invest Ophthalmol Vis Sci 50: 5207-5212, 2009.

4. Ho CS and Giaschi DE: Low- and high-level motion perception deficits in anisometropic and strabismic amblyopia: Evidence from fMRI. Vision Res 49: 2891-2901, 2009.

5. Ho CS, Paul PS, Asirvatham A, Cavanagh P, Cline R and Giaschi DE: Abnormal spatial selection and tracking in children with amblyopia. Vision Res 46: 3274-3283, 2006.

6. Secen J, Culham J, Ho C and Giaschi D: Neural correlates of the multiple-object tracking deficit in amblyopia. Vision Res 51: 2517-2527, 2011.
7. Tripathy SP and Levi DM: On the effective number of tracked trajectories in amblyopic human vision. J Vis 8: 8.1-22, 2008.

8. Sharma V, Levi DM and Klein SA: Undercounting features and missing features: Evidence for a high-level deficit in strabismic amblyopia. Nat Neurosci 3: 496-501, 2000.

9. Popple AV and Levi DM: The attentional blink in amblyopia. J Vis 8: 12.1-9, 2008

10. Farzin F and Norcia AM: Impaired visual decision-making in individuals with amblyopia. J Vis 11: pii: 6, 2011.

11. Thiel A and Sireteanu R: Strabismic amblyopes show a bilateral rightward bias in a line bisection task: Evidence for a visual attention deficit. Vision Res 49: 287-294, 2009.

12. Hou C, Kim YJ, Lai XJ and Verghese P: Degraded attentional modulation of cortical neural populations in strabismic amblyopia. J Vis 16: 16, 2016

13. Ziv N and Omer E: Music and time: The effect of experimental paradigm, musical structure and subjective evaluations on time estimation. Psychol Music 39: 182-195, 2011.

14. Block RA, Hancock PA and Zakay D: How cognitive load affects duration judgments: A meta-analytic review. Acta Psychol (Amst) 134: 330-343, 2010.

15. Arzounian D, de Kerangal $M$ and de Cheveigné A: A sliding two-alternative forced-choice paradigm for pitch discrimination. J Acoust Soc Am 142: 167, 2017.

16. Hagmann-von Arx P, Manicolo O, Perkinson-Gloor N, Weber P, Grob A and Lemola S: Gait in very preterm school-aged children in dual-task paradigms. PLoS One 10: e0144363, 2015.

17. Spekreijse $\mathrm{H}$, Khoe LH and van der Tweel LH: A case of amblyopia; electrophysiology and psychophysics of luminance and contrast. Adv Exp Med Biol 24: 141-156, 1972.

18. Moulden B, Renshaw J and Mather G: Two channels for flicker in the human visual system. Perception 13: 387-400, 1984.

19. Leguire LE, Rogers GL and Bremer DL: Amblyopia: The normal eye is not normal. J Pediatr Ophthalmol Strabismus 27: 32-39, 1990.

20. Xiao M, Wei X, Li Y, Xion W and Xu S: Pattern visual evoked potentials in normal-vision eyes of post-therapy amblyopia. Zhong Nan Da Xue Xue Bao Yi Xue Ban 38: 704-708, 2013 (In Chinese).

21. Giaschi DE, Regan D, Kraft SP and Hong XH: Defective processing of motion-defined form in the fellow eye of patients with unilateral amblyopia. Invest Ophthalmol Vis Sci 33: 2483-2489, 1992

22. Kiorpes L, Tang C and Movshon JA: Sensitivity to visual motion in amblyopic macaque monkeys. Vis Neurosci 23: 247-256, 2006.

23. Ho CS, Giaschi DE, Boden C, Dougherty R, Cline R and Lyons C: Deficient motion perception in the fellow eye of amblyopic children. Vision Res 45: 1615-1627, 2005.

24. Hou F, Huang CB, Tao L, Feng L, Zhou Y and Lu ZL: Training in contrast detection improves motion perception of sinewave gratings in amblyopia. Invest Ophthalmol Vis Sci 52: 6501-6510, 2011.

25. Pianta MJ and Kalloniatis M: Characteristics of anisometropic suppression: Simple reaction time measurements. Percept Psychophys 60: 491-502, 1998

26. Huang PC, Li J, Deng D, Yu M and Hess RF: Temporal synchrony deficits in amblyopia. Invest Ophthalmol Vis Sci 53: 8325-8332, 2012.

27. Noreika V, Falter CM and Rubia K: Timing deficits in attention-deficit/hyperactivity disorder (ADHD): Evidence from neurocognitive and neuroimaging studies. Neuropsycholia 51: 235-266, 2013

28. Hart H, Radua J, Mataix-Cols D and Rubia K: Meta-analysis of fMRI studies of timing in attention-deficit hyperactivity disorder (ADHD). Neurosci Biobehav Rev 36: 2248-2256, 2012.

29. Mioni G, Stablum F and Cantagallo A: Time discrimination in traumatic brain injury patients. J Clin Exp Neuropsychol 35: 90-102, 2013.

30. Li Q, Jiang Q, Guo M, Li Q, Cai C and Yin X: Grey and white matter changes in children with monocular amblyopia: Voxel-based morphometry and diffusion tensor imaging study. Br J Ophthalmol 97: 524-529, 2013. 